\title{
Acute compartment syndrome of the thigh after weight training
}

\author{
J P Bidwell, C E R Gibbons, S Godsiff
}

\begin{abstract}
Compartment syndrome of the thigh is a rare but serious condition that is normally associated with closed trauma or compressive injury. A case of acute compartment syndrome of the thigh occurred in a 16 year old boy after intensive weight training. There was no evidence of muscle tear or focal haemorrhage during subsequent fasciotomy.

(Br F Sports Med 1996;30:264-265)
\end{abstract}

Key terms: compartment syndrome; thigh; fasciotomy; weight training

\section{Case report}

A 16 year old boy presented to casualty complaining of severe pain and swelling in the right thigh. Two days previously, during weight training and without previously warming up, he had performed five minutes of unilateral quadriceps extensions with the right leg, using heavy weights. After the exercise, he felt pain in the anterior aspect of the right thigh, but this worsened abruptly 48 hours later.

At presentation the right thigh was grossly swollen and tender. The circumferential difference between this and the unaffected thigh was $8 \mathrm{~cm}$. There was altered sensation in the outer aspect of the thigh but otherwise the leg was neurologically intact. Passive flexion of the knee exacerbated the pain in the right thigh. Peripheral pulses in the leg were palpable and equal to the left side. Radiographs of the right thigh showed soft tissue swelling, and ultrasound examination revealed diffuse swelling of the muscles of the anterior compartment.

A clinical diagnosis of acute compartment syndrome of the right thigh was made. This was confirmed by measurement of the intracompartmental pressures with a Stryker handheld kit. Measurements of $80 \mathrm{~mm} \mathrm{Hg}$ in the anterior compartment, $35 \mathrm{~mm} \mathrm{Hg}$ in the posterior compartment, and $12 \mathrm{~mm} \mathrm{Hg}$ in the medial compartment were recorded.

The patient was taken to theatre for urgent fasciotomy. The anterior and posterior compartments of the right thigh were decompressed by a single long lateral incision. The anterior compartment was first decompressed by incising the tensor fascia lata. The vastus lateralis was seen to bulge through the wound.
On closer inspection of the anterior compartment the quadriceps muscle appeared oedematous, but no focal haemorrhage or muscle tear could be found. The posterior compartment was decompressed by dividing the lateral intermuscular septum.

The fasciotomy wound was left open and packed, and closed with split-skin grafting five days postoperatively. There was an immediate improvement in his symptoms after fasciotomy, and he made a full recovery once his wounds had healed.

\section{Discussion}

Compartment syndrome has been defined as an increase in the interstitial pressure in a closed osseo-fascial compartment, leading to microvascular compromise of the tissues contained within. ${ }^{1}$ It is a serious condition with considerable morbidity that is seen mainly in the lower leg and forearm, but much less frequently in the thigh and elsewhere.

Aetiological factors of compartment syndrome in the thigh include fractured shaft of femur, vascular injury, external compression of the thigh, and the use of military antishock trousers. ${ }^{2}$ It has also been seen after muscle rupture ${ }^{3}$ and its occurrence after heavy exercise has recently been reported. ${ }^{4}$

The consequences of inadequate treatment are disastrous. In a report from Schwartz et al, ${ }^{2}$ almost $50 \%$ of a group of 21 patients with acute compartment syndrome of the thigh developed crush syndrome (ischaemic muscle necrosis, myoglobinuria, and acute renal failure) and the late sequelae include muscle contractures and distal neurovascular compromise.

In this case of compartment syndrome there was no evidence of intracompartmental haemorrhage or muscle rupture. In a report of 52 patients with acute compartment syndrome in the lower leg due to intense muscle use, ${ }^{5}$ Reneman proposed that the increase in intracompartmental pressure was primarily due to acute muscular hypertrophy, allied with a $20 \%$ increase in volume of skeletal muscle observed in muscle undergoing intensive contraction.

The rarity of compartment syndrome of the thigh is thought to be due the large potential space to allow for swelling and increase in interstitial pressure, and only one other case of 
exercise as an aetiological factor has been reported. ${ }^{4}$

Compartment syndrome of the thigh remains an uncommon finding, but a high index of clinical suspicion should be maintained in the patient with a relevant history and suggestive physical signs. The patient had a painful swollen thigh and passive flexion of the knee caused severe pain in the anterior compartment of the thigh. Compartment pressures can be recorded by the bedside and in this case confirmed a markedly increased pressure in the anterior compartment, with a moderate increase in the posterior compartment. Prompt fasciotomy of both compartments was performed.

Weight training and body building have become increasingly popular forms of sport and recreation. Well supervised training, with particular emphasis on the importance of gentle stretching and warming up, is vital to reduce the multitude of soft tissue injuries that result, and without doubt would have prevented the scenario that we encountered in this case.

1 Mubarak SJ, Hargens SR, Owen CA, Garetto LP, Akeson WH. The wick catheter technique for measurement of WH. The wick catheter technique for measurement of intramuscular pressure: a new research

2 Schwartz JT, Brumback RJ, Lakatos R, Poka A, Bathon GH Burgess AR. Acute compartment syndrome of the thigh. A spectrum of injury. F Bone foint Surg Am 1989;71A:392 400 .

3 Klasson SC, Vander Schilden JL. Acute anterior compartment syndrome of the thigh complicating quadriceps haematoma. Two case reports and review of the literature. Orthop Rev 1990;19:421-7

4 Kahan JSG, McClellan SRT, Burton DS. Acute bilateral compartment syndrome of the thigh induced by exercise. $f$ Bone foint Surg Am 1994;76A:1068-71.

5 Reneman RS. The anterior and lateral compartment

syndrome of the leg due to intensive use of muscles. Clin Orthop 1975;113:69-80.

\title{
Distant entry pneumothorax in a competitive fencer
}

\author{
Peter A Harmer, James Moriarity, Mark Walsh, Michael Bean, Joni Cramer
}

\section{Department of \\ Exercise Science, Willamette University, Salem, Oregon, USA \\ P A Harmer}

University of Notre Dame, South Bend, Indiana, USA

$\mathrm{J}$ Moriarity

St Joseph's Medical Center, South Bend, Indiana, USA

$M$ Walsh

Department of Athletics, University of Notre Dame, South

Bend, Indiana, USA M Bean

California University of Pennsylvania, California, Pennsylvania, USA J Cramer

Correspondence to: Dr Peter A Harmer, Department of Exercise Science-Sports Medicine, Willamette University, Salem, OR 97301, USA.

Accepted for publication 16 January 1996

\begin{abstract}
An elite level fencer sustained a penetrating wound to the upper arm after his opponent's blade broke. Standard care for a deep puncture wound was given but it was some time before the athlete presented symptoms of a pneumothorax, which was confirmed by radiograph. Although resolution of this case was unremarkable, the possibility of penetrating thoracic injury, even when the point of entry is well outside the thorax and the athlete is not immediately symptomatic, should be born in mind by medical personnel working with fencers.

(Br $\mathcal{F}$ Sports Med 1996;30:265-266)
\end{abstract}

Key terms: fencing; penetrating wound; pneumothorax

Pneumothorax is an uncommon injury in noncontact sports. Fencing, although defined as a non-contact sport, involves fast and powerful blade contact with various parts of an opponent's body (principally the torso). Despite its heritage of lethal intent, there is little evidence to indicate that fencing is a hazardous activity. ${ }^{1-3}$ However, there is an inherent risk of serious injury especially when blades break, because of the probability of lacerations or penetrating wounds from rigid remnants. The thoracic wall (right side for right handed fencers; left for left handed fencers) is particularly vulnerable. It is left exposed as fencers align themselves in the typical en guarde position, that is, along the frontal plane with the fencing arm abducted, exposing the axilla and lateral thoracic wall to the opponent's blade. Incidents of penetrating wounds are well known to competitive fencers but are not well documented in published reports in general medical or sports medicine journals. The present case is an example of a complex penetrating wound involving a pneumothorax from a broken blade.

\section{Case report}

A right handed elite level American fencer was competing in an epee tournament. During a thrusting manoeuvre his opponent's blade struck the guard on the victim's weapon and was fractured. The remnant of the blade (still attached to the opponent's handle) continued its forward momentum scraping the volar surface of the victim's right arm penetrating it at a point $12 \mathrm{~cm}$ distal to the anterior axillary line (fig 1). The fencer experienced an unusual discomfort in his back but remained unaware of his injury until a large amount of blood became evident on the upper sleeve of his outer garment. The match was discontinued and the wound was inspected and a compression bandage applied. Chest pain was noted to be present, specifically when the fencer was lying flat. Vital signs were normal and chest auscultation revealed equal breath sounds. The fencer was transported to hospital for further evaluation. In the emergency department the fencer continued to experience chest discomfort remarkably similar to his remembered asthma attacks as a child. Breath sounds were noted to be slightly diminished on the right side and a chest radiograph was requested. 